\title{
Worth the wait: effects of age of onset of marijuana use on white matter and impulsivity
}

\author{
Staci A. Gruber • Mary Kathryn Dahlgren • \\ Kelly A. Sagar • Atilla Gönenç • Scott E. Lukas
}

Received: 10 July 2013 / Accepted: 8 October 2013 / Published online: 5 November 2013

(C) The Author(s) 2013. This article is published with open access at Springerlink.com

\begin{abstract}
Rationale Marijuana (MJ) use continues to rise, and as the perceived risk of using $\mathrm{MJ}$ approaches an all-time historic low, initiation of MJ use is occurring at even younger ages. As adolescence is a critical period of neuromaturation, teens and emerging adults are at greater risk for experiencing the negative effects of MJ on the brain. In particular, MJ use has been shown to be associated with alterations in frontal white matter microstructure, which may be related to reports of increased levels of impulsivity in this population.

Objectives The aim of this study was to examine the relationship between age of onset of MJ use, white matter microstructure, and reported impulsivity in chronic, heavy MJ smokers.

Methods Twenty-five MJ smokers and 18 healthy controls underwent diffusion tensor imaging and completed the Barratt Impulsiveness Scale. MJ smokers were also divided into early onset (regular use prior to age 16) and late onset (age 16 or later) groups in order to clarify the impact of age of onset of MJ use on these variables.

Results MJ smokers exhibited significantly reduced fractional anisotropy (FA) relative to controls, as well as higher levels of impulsivity. Earlier MJ onset was also associated with lower
\end{abstract}

S. A. Gruber $(\bowtie) \cdot$ M. K. Dahlgren • K. A. Sagar • A. Gönenç

Cognitive and Clinical Neuroimaging Core,

McLean Imaging Center, McLean Hospital,

115 Mill St, Belmont, MA 02478, USA

e-mail: gruber@mclean.harvard.edu

S. A. Gruber · A. Gönenç · S. E. Lukas

Department of Psychiatry, Harvard Medical School,

Boston, MA 02215, USA

\section{S. E. Lukas}

Behavioral Psychopharmacology Research Laboratory,

McLean Imaging Center, McLean Hospital,

115 Mill St, Belmont, MA 02478, USA levels of FA. Interestingly, within the early onset group, higher impulsivity scores were correlated with lower FA, a relationship that was not observed in the late onset smokers. Conclusions MJ use is associated with white matter development and reported impulsivity, particularly in early onset smokers.

Keywords Marijuana - Age of onset - White matter . Diffusion tensor imaging $\cdot$ Impulsivity

\section{Introduction}

Numerous studies report impairment in executive function, specifically behavioral response inhibition, in individuals with marijuana use disorders (Pope and Yurgelun-Todd 1996; Bolla et al. 1999; Gruber et al. 2011a); however, current dialogues regarding legalization of marijuana (MJ) and the use of medical MJ have likely reduced concern regarding the potential negative impact of MJ on cognitive processes. Within the USA, MJ remains the most widely used illicit substance, with an estimated 18.1 million past-month users; $80.5 \%$ of current illicit drug users report using marijuana, and $64.3 \%$ report that it is the only drug they use (SAMHSA 2012). In 2012, Colorado and Washington became the first states to legalize MJ without medical constraints; 2012 also brought a record of 18 states with legalized medical MJ (ONDCP 2012). As current deliberations over the legalization of MJ often highlight the benefits of medical MJ, it is perhaps not surprising that the perceived risk of MJ has reached an alltime historic low among the nation's emerging adults; the most recent Monitoring the Future Study reported that fewer than half of high school seniors said they thought regular MJ smoking was harmful (Johnston et al. 2012). Accompanying the increase in MJ use overall, the average age of first use is now significantly lower than in previous years, dropping from 
17.8 years to 17 . As adolescence is a time of neuromaturation, with increasing evidence that the adolescent brain is more vulnerable to the effects of drugs than the adult brain (Monti et al. 2005), those at the greatest risk for adverse consequences represent a growing population of consumers of MJ (Schneider 2008), posing a serious public health concern.

In addition to findings that suggest alterations in neurocognitive function (Pope and Yurgelun-Todd 1996; Harvey et al. 2007; McHale and Hunt 2008; Solowij and Pesa 2010; Gruber et al. 2011a; Lisdahl and Price 2012) and inhibitory processing (Gruber and Yurgelun-Todd 2005; Tapert et al. 2007; Schweinsburg et al. 2008; Jacobus et al. 2009a; Gruber et al. 2012a) in chronic MJ smokers relative to control participants, several investigations have also reported alterations in frontal white matter microstructure, measured with diffusion tensor imaging (DTI) techniques in adult MJ smokers (Arnone et al. 2008; Ashtari et al. 2009; Bava et al. 2009; Jacobus et al. 2009b; Gruber et al. 2011b). Arnone et al. (2008) reported increased mean diffusivity (MD)/trace in the prefrontal area of the corpus callosum in MJ relative to controls; this trended towards a positive correlation between diffusivity levels and length of use. Similarly, Ashtari et al. (2009) used voxelwise and tractography techniques and reported reduced fractional anisotropy (FA), a measure of directionality and coherence in white matter fibers, and increased trace in frontotemporal regions in MJ smokers relative to nonsmoking controls. Adolescent studies report similar findings; Bava et al. (2009) highlighted decreases in FA in MJ and alcohol-using adolescents relative to controls in both inferior frontal and temporal areas. Jacobus et al. (2009b) reported reduced white matter in MJ-smoking adolescents who were also binge drinkers relative to controls, but who had higher FA values relative to pure binge drinkers, suggesting a potential neuroprotective effect of MJ, perhaps due to reducing alcohol-related oxidative stress. Finally, a recent study from our group found significant reductions in frontal FA in chronic MJ smokers relative to control participants, which was positively correlated with age of onset of MJ use (Gruber et al. 2011b). Overall, these studies suggest altered white matter integrity in $\mathrm{MJ}$ smokers relative to control participants and underscore the importance of examining the relationship between age of onset of MJ use and white matter measures, as early exposure may result in enduring structural brain changes.

Impulsivity is a complex and multidimensional construct and is well documented in individuals with substance use problems (Brady et al. 1998; Vitaro et al. 1998; Heil et al. 2006). Higher levels of both self-reported impulsivity, as measured by the Barratt Impulsiveness Scale (BIS), and risk taking have previously been reported in some studies of substance abusers (Lejuez et al. 2002; Lejuez et al. 2003; Gruber and Yurgelun-Todd 2005). Further, an impulsive personality style has previously been identified as both a risk factor and predictor of substance abuse and dependence (Guy et al. 1994). Interestingly, few studies thus far have focused on the specific relationship between reported or observed impulsivity and MJ use. Vangsness et al. (2005) examined the role of positive and negative MJ-related expectancies in MJ users and reported that individuals with higher levels of impulsivity held fewer negative expectancies related to MJ and, in turn, used MJ more often than those with lower levels of impulsivity. In a more recent study of delay discounting, a behavioral index of impulsivity, Johnson et al. (2010) reported that $\mathrm{MJ}$-dependent participants demonstrated a trend towards increased delay discounting relative to controls and former MJ smokers. Further, dependent MJ users scored significantly higher than controls on the impulsiveness subscale of the Eysenck Impulsiveness-Venturesomeness-Empathy questionnaire. Taken together with the neuroimaging findings of MJ smokers that report alterations in response inhibition as well as decision making (Gruber and Yurgelun-Todd 2005; Tapert et al. 2007; Schweinsburg et al. 2008; Jacobus et al. 2009a; Gruber et al. 2012a), impulsivity may, in fact, reflect a stable trait in those who smoke MJ relative to those who do not.

DTI studies of substance abusers have revealed that reductions in FA are associated with impulsivity (Lim et al. 2002; Moeller et al. 2005; Gruber et al. 2011b). Lim et al. (2002) reported reductions in frontal FA in cocaine users relative to controls, suggesting cocaine dependence involves disruptions of orbitofrontal connectivity, which is critical for decision making. Moeller et al. (2005) reported reduced FA in both the genu and rostral body of the anterior corpus callosum in cocaine-dependent participants, as well as increased BIS scores and errors of impulsivity on a continuous performance task relative to controls. The authors concluded that reductions in white matter coherence were associated with reported and observed impulsivity and are consistent with prior theories regarding frontal cortical involvement in impaired inhibitory control in cocaine dependence. These findings and our own (Gruber et al. 2011b) lend support to the growing hypothesis that substance abuse is related to white matter microstructural changes, which are associated with compromised ability to perform tasks requiring cognitive control and greater difficulty regulating impulse control.

In order to better understand the relationship among MJ use, white matter microstructure, and self-reported impulsivity, we examined data from chronic, heavy MJ smokers and healthy control participants who did not smoke MJ. We hypothesized that relative to control subjects, MJ smokers would have lower white matter fiber tract integrity, as measured by FA, a measure of directionality and coherence/ organization of white matter fibers; higher MD, the average of diffusion in multiple directions; and higher levels of reported impulsivity. Further, we predicted that early onset MJ smokers would drive the finding between the non-MJ-smoking controls and MJ smokers, with earlier age of onset associated 
with higher reported impulsivity and lower white matter organization.

\section{Methods}

Participants

Participants were recruited from the Greater Boston Metropolitan area and included 25 chronic, heavy MJ smokers and 18 healthy control participants who did not smoke MJ. All participants were administered the Structured Clinical Interview for DSM-IV, Patient Edition (SCID-P; First et al. 1994) to ensure that no Axis I pathology was present other than MJ use in the smoking group. In addition, to qualify for enrollment, MJ smokers had to have reported smoking a minimum of 2,500 times, used MJ at least 5 out of the last 7 days, tested positive for urinary cannabinoids, and met DSM-IV criteria for MJ abuse or dependence. MJ smokers were also required to abstain from smoking at least $12 \mathrm{~h}$ before their study visit in order to ensure that they were not acutely intoxicated at the time of assessment. All participants were required to provide a urine sample upon arrival at the laboratory, and in order to ensure adherence to the 12-h abstinence schedule, MJ participants were led to believe that our researchers could use this sample to detect the last use of MJ within this time frame, a method we have successfully utilized in the past (Gruber et al. 2012a; Gruber et al. 2012b; Gruber et al. 2011a). Participants, however, were excluded from this study if they reported more than 15 lifetime uses of any category of illicit drugs or recreational use of prescription drugs or had a positive urine screen for any drug (excluding MJ for the smoking group). Individuals were also not enrolled if they reported any head injury with loss of consciousness, a history of any neurological disorder, or previous use of psychotropic medications.

Prior to participation, study procedures were explained, and all participants were required to read and sign an informed consent form approved by the McLean Hospital Institutional Review Board, which described the procedures and voluntary nature of the study.

\section{Study design}

Marijuana use was assessed using a modified timeline followback procedure. Participants provided information regarding history of MJ use, including age of onset, and duration of use (years), as well as current frequency (smokes per week), magnitude (grams per week), and mode of use. Lifetime use was also determined via the SCID-P. In order to explore the potential impact of age of onset on reported impulsivity and white matter alterations, the MJ smokers were further divided into two groups based on age of onset of regular MJ use. For this study, age of "regular" use of MJ was defined as the age at which subjects began using MJ on a routine, expected, and consistent basis and not the age at which they "tried" MJ for the first time. Early onset was defined as regular MJ use prior to age $16(n=11)$ while and late onset was defined as first regular MJ use at age 16 or later $(n=14)$.

In order to assess self-reported impulsivity, all participants completed the Barratt Impulsiveness Scale (BIS 11; Patton et al. 1995), which provides subscales for three domains, including attention, motor, and non-planning, as well as a total impulsiveness score. Although the BIS 11 has been considered a gold standard in self-report assessments of impulsivity, recent work has questioned the utility of the original, three-factor model from which scores are derived. In order to compare the validity of the BIS 11 results obtained using the standard threescore reporting method (Patton et al. 1995), we also utilized two alternative scoring methods, the BIS-Brief as described by Steinberg et al. (2013) which provides a condensed, unidimensional representation of self-reported impulsivity, as well as the Reise et al. (2013) model, which provides a twofactor model of cognitive and behavioral impulsivity. Participants also completed several self-report questionnaires in order to ensure that groups were well matched for clinical state at the time of scanning. Briefly, self-report measures included the Profile of Mood States (Pollock et al. 1979), which provides a profile of current mood state for the individual domains of vigor, anger, confusion, tension, and depression, as well as a total mood disturbance score; the Beck Depression Inventory (Beck et al. 1961); and the State-Trait Anxiety Inventory (Spielberger et al. 1983). Further, participants also completed the Positive and Negative Affect Scale (Watson et al. 1988), which provides a score for both positive and negative affect; the Montgomery-Asberg Depression Rating Scale (Montgomery and Asberg 1979); the Hamilton Anxiety Scale (Hamilton 1959); and the Young Mania Rating Scale (Young et al. 1978). In addition, participants completed the four-factor Wechsler Abbreviated Scale of Intelligence, which provides a measure of general intellectual function and yields an estimate of IQ (Wechsler 1999).

Imaging methods

As part of a larger neuroimaging study, participants completed DTI, which was performed on a Siemens Trio whole-body 3-T MRI scanner (Siemens Corporation, Erlangen, Germany) using a 12-channel, phased array head coil. Data was acquired in the axial plane using a diffusion-weighted standard single shot, double spin echo, echo planar protocol with the following parameters: TR $/ \mathrm{TE}=9.3 \mathrm{~s} / 89 \mathrm{~ms}$; matrix $=128 \times 128$ on a 25.6 $\mathrm{cm}$ FOV; slice thickness $=2 \mathrm{~mm}$ with a gap of 0 ; and $b$ value $=$ $700 \mathrm{~s} / \mathrm{mm}^{2}$, for a total acquisition time of $8 \mathrm{~min}$ and $41 \mathrm{~s}$. Diffusion was measured along 48 noncollinear directions (number of exCitations $=1$ ). For each slice, seven images with no diffusion weighting $\left(b=0 \mathrm{~s} / \mathrm{mm}^{2}\right)$ were also acquired. 
Image processing and analysis

All processing of the diffusion-weighted images was performed using the FMRIB Software Library (version 4.1.9; http://www. fmrib.ox.ac.uk/fsl). Images were corrected for motion and eddy current to remove nonlinear artifacts and distortion from the datasets by applying affine alignment of each diffusionweighted image to the first volume of the diffusion data without gradient $(b=0)$. A binary mask was generated from $b=0$ image using the Brain Extraction Tool and tensors were fit using the $b$ factor and diffusion direction matrix. Eigenvalues and eigenvectors were calculated for each voxel resulting in diffusion-weighted scalar maps, including whole brain FA and MD. These scalar maps were then aligned to standard space using the steps described for Tract-Based Spatial Statistics (TBSS), also part of the FSL package. Finally, FA and $\mathrm{MD}$ values were extracted in the anatomical regions defined by the John Hopkins University White Matter Label Atlas by registering the white matter labels to subject space using the inverse of the transform previously used to register subject data to standard space. In order to assess white matter differences in regions previously examined (Gruber et al. 2011b), we limited our selection of atlas labels to the following bilateral brain regions: genu of the corpus callosum, corona radiata, and internal and external capsule.

\section{Results}

As noted in Table 1, participants were well matched and did not differ significantly with regard to age, IQ, days of alcohol use, and socioeconomic status. In order to assess cigarette smoking/nicotine use, all subjects completed the Fagerstrom Test for Nicotine Dependence (FTND), and scores did not differ significantly between the groups (see Table 1). However, as some previous studies have noted a relationship between white matter measures and cigarette smoking (Jacobsen et al. 2007; Paul et al. 2008; Hudkins et al. 2012), we also assessed how many individuals in both of the study groups used cigarettes and completed a chi-square analysis. The chi-square analysis revealed no significant difference in the observed frequency of current cigarette smokers between the control and MJ groups $\left(X^{2}(1, N=43)=2.61\right)$.

It is of note that regardless of the scoring method used for the BIS 11, MJ smokers had significantly higher self-reported impulsivity scores relative to the healthy control participants (see Table 2). As a result, and in order to remain comparable with previous work, all additional analyses were completed using only those scores derived from the standard three-factor scoring method. Each of the three BIS subscores and the total score reached statistical significance: attention $(t(41)=1.89$, $p=0.03)$, motor $(t(41)=1.91, p=0.03)$, non-planning $(t(41)=$ $1.92, p=0.03)$, and total impulsivity $(t(41)=2.28, p=0.01)$ scores (Table 2).

Comparisons of early and late onset smokers revealed no significant differences in patterns of MJ use between the two groups. However, it is of note that early onset smokers smoked slightly more often (smokes/week; 18.76 vs. 15.51) and more than twice as much MJ per week (grams/week; 14.65 vs. 6.66) as late onset smokers (see Table 3).

Analyses of the DTI data which focused on regions we have previously explored (Gruber et al. 2011b) revealed significantly reduced FA in MJ smokers relative to controls in several regions of interest, including the left $(t(41)=2.10$, $p=0.02)$ and right genu of the corpus callosum $(t(41)=2.28$, $p=0.01)$ and the left internal capsule $(t(41)=1.71, p=0.05)$. Trends for reduced FA were also observed in the right internal capsule $(t(41)=1.40, p=0.09)$ as well as the left $(t(41)=1.52$, $p=0.07)$ and right $(t(41)=1.53, p=0.07)$ external capsule. In addition to reduced FA, mean diffusivity (often inversely correlated with FA values) was also significantly higher in MJ smokers relative to controls in the genu (left: $t(41)=1.99$, $p=0.03$; right: $t(41)=2.11, p=0.02$ ) (see Table 4). When divided into early and late MJ smokers, no significant differences in FA were noted between the groups. It is of note, however, that correlation analyses of the entire MJ group which examined the association between age of MJ onset and FA revealed that earlier age of onset was related to lower

Table 1 Participant demographics

\begin{tabular}{llll}
\hline Demographics & Healthy controls & MJ smokers & $T$ test (2-tailed) \\
\hline$N$ & $18(7 \mathrm{M}, 11 \mathrm{~F})$ & $25(18 \mathrm{M}, 7 \mathrm{~F})$ & - \\
Handedness & $17 \mathrm{R}, 1 \mathrm{~L}$ & $24 \mathrm{R}, 1 \mathrm{~L}$ & - \\
Age & $23.11 \pm 3.51$ & $23.16 \pm 5.87$ & $\mathrm{NS}$ \\
VIQ & $127.39 \pm 5.84$ & $120.09 \pm 17.83$ & $\mathrm{NS}$ \\
PIQ & $117.22 \pm 11.49$ & $114.50 \pm 9.42$ & $\mathrm{NS}$ \\
FTND & $0.00 \pm 0.00$ & $0.39 \pm 0.99$ & $\mathrm{NS}$ \\
Days of alcohol use in past 30 & $4.61 \pm 4.60$ & $7.24 \pm 6.20$ & $\mathrm{NS}$ \\
Hollingshead 4-factor SES index & $49.67 \pm 10.85$ & $50.04 \pm 10.98$ & $\mathrm{NS}$ \\
\hline
\end{tabular}

$V I Q$ verbal IQ, PIQ performance IQ, FTND Fagerstrom test for nicotine dependence, SES socioeconomic status 
Table 2 Barratt Impulsivity Scale (BIS-11) scores

\begin{tabular}{lllll}
\hline $\begin{array}{l}\text { BIS-11 scoring } \\
\text { method }\end{array}$ & $\begin{array}{l}\text { Healthy } \\
\text { controls }\end{array}$ & $\begin{array}{l}\text { MJ } \\
\text { smokers }\end{array}$ & $p$ (1-tailed) & $\begin{array}{l}\text { Effect } \\
\text { size }\left(\eta^{2}\right)\end{array}$ \\
\hline Patton & & & & \\
$\quad$ Attention & $14.58 \pm 4.42$ & $16.83 \pm 3.40$ & 0.033 & 0.080 \\
$\quad$ Motor & $19.90 \pm 3.99$ & $22.54 \pm 4.80$ & 0.032 & 0.081 \\
$\quad$ Non-planning & $21.14 \pm 5.72$ & $24.02 \pm 4.10$ & 0.031 & 0.083 \\
$\quad \begin{array}{l}\text { Total } \\
\text { Reise }\end{array}$ & $55.76 \pm 12.34$ & $63.50 \pm 9.87$ & 0.014 & 0.113 \\
$\quad$ Cognitive & $11.22 \pm 3.67$ & $12.92 \pm 2.71$ & 0.044 & 0.069 \\
$\quad$ Behavioral & $11.99 \pm 3.09$ & $14.19 \pm 3.65$ & 0.022 & 0.095 \\
$\begin{array}{l}\text { Steinberg } \\
\quad \text { Brief }\end{array}$ & $14.79 \pm 4.44$ & $17.01 \pm 3.29$ & 0.034 & 0.079 \\
\hline
\end{tabular}

levels of FA in the left genu $(r(23)=.344, p=0.05)$ and right genu $(r(23)=.358, p=0.04)$; see Fig. 1 .

In order to explore the possible relationship between reported impulsivity and white matter alterations, we examined correlations between BIS scores and levels of FA within the MJ smokers. Analyses revealed that FA in the right genu was significantly inversely correlated with BIS attention $(r(23)=$ $-0.500, p=0.01)$ and motor $(r(23)=-0.337 ; p=0.05)$, and a trend was noted for BIS total impulsiveness scores $(r(23)=$ -0.292; $p=0.08$ ); lower FA levels were associated with higher levels of impulsivity. Similarly, left genu FA was negatively correlated with BIS attention scores $(r(23)=-0.372, p=0.03)$. Interestingly, when the MJ-smoking group was separated into those with early vs. late onset, it appeared that these relationships were driven almost exclusively by the early onset smokers, as higher impulsivity scores on all BIS subscales were significantly associated with lower FA in both the left and right genu of early onset smokers (see Fig. 2).

\section{Discussion}

As hypothesized, we found significantly reduced FA in both left and right genu of the corpus callosum and significantly higher BIS scores for the MJ smokers relative to the non-MJsmoking control participants. Correlation analyses revealed a significant relationship between the age of MJ onset and FA in both the left and right genu, suggesting that earlier onset of MJ use is associated with lower white matter fiber tract integrity in these brain regions. Further, while BIS scores were inversely correlated with FA in the MJ smokers, with lower levels of FA associated with higher levels of reported impulsivity, this relationship was driven primarily by the early onset smoking group; higher impulsivity scores were significantly associated with lower FA in both the left and right genu of early onset smokers. In the aggregate, these data suggest that a specific relationship exists between FA and behavioral impulsivity in those who begin smoking MJ prior to the age of 16 .

Data from the present study are consistent with previous studies of white matter microstructure in MJ smokers (Arnone et al. 2008; Ashtari et al. 2009; Bava et al. 2009; Jacobus et al. 2009a, 2009b), as well as studies that have independently examined impulsivity in substance-abusing populations (Vangsness et al. 2005; Johnson et al. 2010). Specifically, previous studies have reported decreased FA, as well as higher levels of behavioral impulsivity in MJ smokers. Results from a recent study of the effects of MJ use on white matter integrity suggest that impaired axonal connectivity exists in several regions of the corpus callosum in heavy MJ users (Zalesky et al. 2012). In addition, the authors report that age of onset of MJ was a significant factor in determining the severity of microstructural alteration; both radial and axial diffusivity were correlated with age of MJ onset. The relationship noted between altered white matter measures and age of onset may be the result of the development of white matter in the adolescent brain. During these years, age appropriate myelination is represented by progressive increases in FA and decreases in mean diffusivity (Morriss et al. 1999), which occurs in parallel with the process of cognitive development, most notably improvements in executive function. Early exposure to MJ during a vulnerable period of development may therefore result in lasting morphologic changes as evidenced in animal (Cha et al. 2006; Schneider and Koch, 2003) and human (Yucel et al. 2008; Mata et al. 2010) studies.

Recent investigations also lend support to our finding of increased behavioral impulsivity in MJ smokers. Dougherty et al. (2013) reported significantly higher BIS scores in adolescent MJ smokers relative to non-MJ-smoking controls. Further, MJ smokers demonstrated deficits in several neurocognitive domains, including those related to impulse control. Difficulty with inhibition and impulse control are likely to have consequences in the daily lives of MJ smokers, and in
Table 3 MJ use characteristics: early vs late onset

\begin{tabular}{llll}
\hline MJ-related variables & Early MJ onset $(n=11)$ & Late MJ onset $(n=14)$ & $T$ test (2-tailed) \\
\hline Age of MJ onset & $14.46 \pm 0.69$ & $17.93 \pm 2.13$ & $<0.01$ \\
Smoking episodes/week & $18.76 \pm 9.38$ & $15.51 \pm 7.19$ & $\mathrm{NS}$ \\
Grams/week & $14.65 \pm 18.97$ & $6.66 \pm 5.56$ & $\mathrm{NS}$ \\
Duration of MJ use (years) & $8.82 \pm 5.67$ & $5.14 \pm 4.42$ & $\mathrm{NS}$ \\
Urinary THC concentration & $405.67 \pm 318.36$ & $743.74 \pm 1091.07$ & $\mathrm{NS}$ \\
\hline
\end{tabular}


Table 4 Mean fractional anisotropy and mean diffusivity in MJ smokers and controls

\begin{tabular}{lllll}
\hline Fractional anisotropy (FA) & Healthy controls & MJ smokers & $T$ test (1-tailed) & Effect size $\left(\eta^{2}\right)$ \\
\hline Left genu & $4.67 \mathrm{E}-1 \pm 0.67 \mathrm{E}-1$ & $4.23 \mathrm{E}-1 \pm 0.67 \mathrm{E}-1$ & 0.02 & 0.097 \\
Right genu & $4.77 \mathrm{E}-1 \pm 0.59 \mathrm{E} 1$ & $4.31 \mathrm{E}-1 \pm 0.68 \mathrm{E}-1$ & 0.01 & 0.113 \\
Left corona radiata & $3.86 \mathrm{E}-1 \pm 0.71 \mathrm{E}-1$ & $3.65 \mathrm{E}-1 \pm 0.65 \mathrm{E}-1$ & NS & - \\
Right corona radiata & $3.86 \mathrm{E}-1 \pm 0.67 \mathrm{E}-1$ & $3.61 \mathrm{E}-1 \pm 0.64 \mathrm{E}-1$ & NS & - \\
Left internal capsule & $4.76 \mathrm{E}-1 \pm 0.70 \mathrm{E}-1$ & $4.44 \mathrm{E}-1 \pm 0.53 \mathrm{E}-1$ & 0.05 & 0.066 \\
Right internal capsule & $4.68 \mathrm{E}-1 \pm 0.55 \mathrm{E}-1$ & $4.43 \mathrm{E}-1 \pm 0.59 \mathrm{E}-1$ & 0.09 & 0.045 \\
Left external capsule & $3.39 \mathrm{E}-1 \pm 0.43 \mathrm{E}-1$ & $3.21 \mathrm{E}-1 \pm 0.37 \mathrm{E}-1$ & 0.07 & 0.053 \\
Right external capsule & $3.38 \mathrm{E}-1 \pm 0.44 \mathrm{E}-1$ & $3.20 \mathrm{E}-1 \pm 0.32 \mathrm{E}-1$ & 0.07 & 0.054 \\
& & & & \\
Mean diffusivity (MD) & & & & 0.088 \\
& & & 0.098 \\
Left genu & $1.07 \mathrm{E}-3 \pm 0.24 \mathrm{E}-3$ & $1.22 \mathrm{E}-3 \pm 0.24 \mathrm{E}-3$ & 0.03 & - \\
Right genu & $1.08 \mathrm{E}-3 \pm 0.29 \mathrm{E}-3$ & $1.26 \mathrm{E}-3 \pm 0.26 \mathrm{E}-3$ & 0.02 & - \\
Left corona radiata & $8.47 \mathrm{E}-4 \pm 1.30 \mathrm{E}-4$ & $8.68 \mathrm{E}-3 \pm 1.30 \mathrm{E}-4$ & NS & - \\
Right corona radiata & $8.71 \mathrm{E}-4 \pm 1.36 \mathrm{E}-4$ & $8.68 \mathrm{E}-3 \pm 1.13 \mathrm{E}-4$ & NS & - \\
Left internal capsule & $8.72 \mathrm{E}-4 \pm 1.55 \mathrm{E}-4$ & $8.91 \mathrm{E}-3 \pm 1.40 \mathrm{E}-4$ & NS & - \\
Right internal capsule & $8.95 \mathrm{E}-4 \pm 1.45 \mathrm{E}-4$ & $8.70 \mathrm{E}-3 \pm 1.25 \mathrm{E}-4$ & NS & - \\
Left external capsule & $9.04 \mathrm{E}-4 \pm 1.28 \mathrm{E}-4$ & $9.32 \mathrm{E}-3 \pm 1.31 \mathrm{E}-4$ & NS & \\
Right external capsule & $8.93 \mathrm{E}-4 \pm 1.23 \mathrm{E}-4$ & $8.91 \mathrm{E}-3 \pm 1.04 \mathrm{E}-4$ & NS & \\
\hline
\end{tabular}

fact, results of a recent study of MJ-related problems in frequent users revealed that higher trait impulsivity scores predicted a greater number of problems in MJ users, most commonly procrastination and lower energy levels (Day et al. 2013).

While several studies have independently investigated white matter alterations and impulsivity, the current study was focused on the specific association between alterations in white matter microstructure and impulsivity in MJ smokers, a relationship previously detected in other substance-abusing populations, including cocaine-dependent subjects (Moeller et al. 2005) and methamphetamine abusers (Salo et al. 2009). Further, the current investigation analyzed the impact of age of onset of MJ use on these variables, which revealed that decreased white matter integrity is associated with higher levels of impulsivity, specifically among individuals who initiated MJ use prior to age 16 . This specific relationship, noted primarily in the early onset smokers, is perhaps related to the changing distribution of cannabinoid receptors, which occurs throughout the normal trajectory of white matter development during adolescence. Research in both animals and humans has suggested that the developing nervous system contains an abundance of cannabinoid receptors in neural fiber tracts, which diminishes over time and becomes redistributed heterogeneously throughout the adult brain (Romero et al. 1997; Glass et al. 1998). Higher concentrations of cannabinoid receptors in neural fiber tracts during adolescence may therefore represent a period of specific vulnerability to the effects of $\mathrm{MJ}$ use on white matter microstructure, which is of particular concern given the importance of frontal regions for the successful completion of tasks requiring cognitive control and inhibition. In a recent study of healthy children aged 7-9, Chaddock-Heyman et al. (2013) found a significant
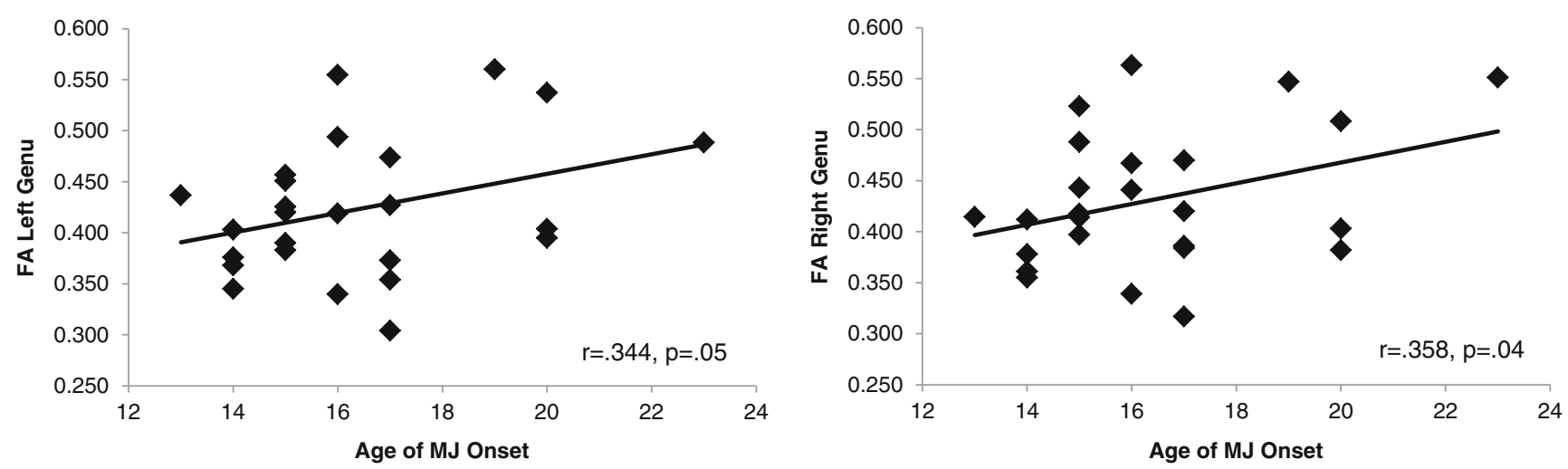

Fig. 1 Correlational analyses of age of onset of MJ use and left and right genu FA 

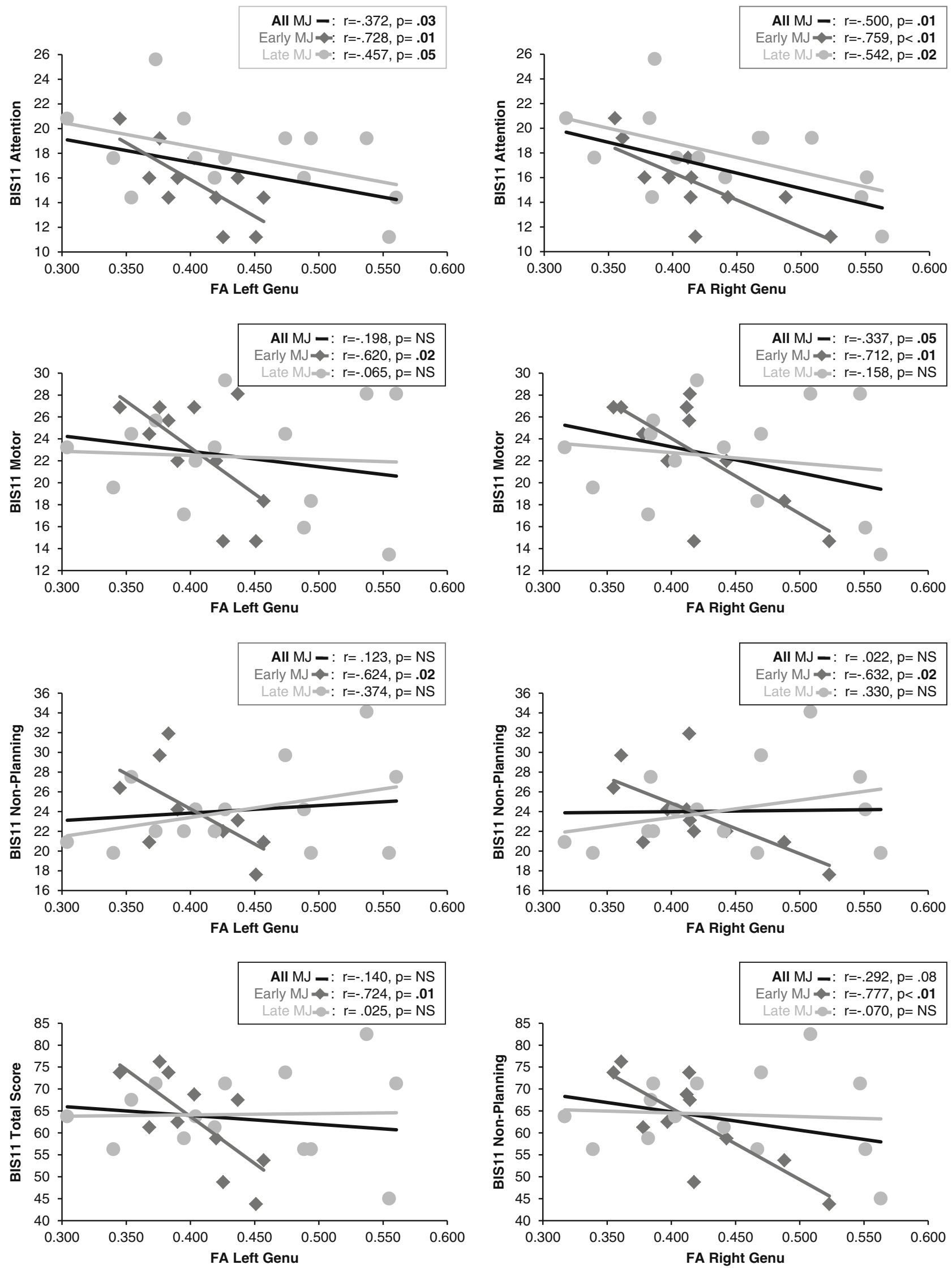

Fig. 2 Correlational analyses of BIS scores vs left and right genu FA 
relationship between greater white matter integrity and better performance on trials of a task requiring cognitive control. It is not surprising, therefore, that chronic, heavy MJ smokers, specifically those who began smoking prior to age 16, have been shown to exhibit both white matter alterations and a compromised ability to inhibit inappropriate responses on cognitive measures (Gruber et al. 2012a).

The current finding of higher reported impulsivity scores which are associated with white matter alterations in $\mathrm{MJ}$ smokers may also be related to the crossing of fibers through the genu, connecting the left and right dorsolateral prefrontal cortex (DLPFC), which has strong interconnections to the anterior cingulate cortex (ACC; Pandya and Seltzer 1982; Park et al. 2008). Both the ACC and DLPFC are components of the cingulo-fronto-parietal cognitive attention network, which is implicated in executive control, inhibition, attention, and feedback-based decision making (Bush et al. 2008). Decreased FA in the genu of MJ smokers relative to control participants may therefore alter this network, resulting in the difficulties observed in MJ smokers with impulse control. This network may also be particularly susceptible to the effects of long-term exposure of MJ, as the fibers within the genu are thinner than those in other regions, such as the splenium (Aboitiz et al. 1996).

\section{Limitations}

While findings from this investigation are compelling, a number of limitations should be noted. The current study included a limited sample size, and future studies should expand research in this area by utilizing larger study samples. Further, in the current study, a few individuals in both the MJsmoking and healthy comparison group reported having used cigarettes, albeit at extremely low levels. Given the potential impact of cigarette smoking on brain structure, future studies should include only individuals who are naïve to cigarette or nicotine use. It is of note that no significant differences in FTND scores or in the number of individuals who used smoked cigarettes or used nicotine (past or present) were detected between the groups in the current study, making it unlikely that the between differences were impacted by cigarettes or nicotine. However, given the fact that slightly more MJ smokers smoked cigarettes than healthy control subjects (although this difference was not statistically significant), and that recent research has shown higher FA in cigarette smokers relative to nonsmokers (Jacobsen et al. 2007; Paul et al. 2008; Hudkins et al. 2012), the inclusion of infrequent cigarette smokers in the currently investigation likely only would have underestimated between group differences in FA.

In addition, previous studies, including our own (Gruber et al. 2011b), have reported frontal white matter alterations in MJ smokers. Although the current study reports decreased FA in MJ smokers and is consistent with our previous work in this area, we previously reported a positive correlation between BIS scores and frontal FA. It is of note, however, that the previous investigation included a more limited DTI scheme, acquiring data from only six directions, and only a subset of subjects $(N=$ 10 ) had completed both the BIS and DTI measures. In contrast, the present study utilized a more comprehensive DTI acquisition method (48 directions), advanced standardized analytic techniques (TBSS), and a significantly larger sample size, which provides confidence in the current results.

While all of our MJ-smoking participants were classified as chronic, heavy smokers and were required to smoke daily or a minimum of 5 of the last 7 days, none of those included in the present study met diagnostic criteria for MJ dependence, while all met for MJ abuse. Study findings may therefore be specific to individuals who do not endorse the more negative effects of marijuana use (i.e., psychological issues, inability to stop or cut down on use, withdrawal effects) and to those who do not meet for dependence, despite frequent, heavy use. It is of note, however, that none of the MJ-smoking participants in the current investigation were seeking treatment and would therefore not likely have come to the attention of a health care provider, yet they demonstrated significant differences in measures of white matter organization and impulsivity. Taken together with the growing numbers of emerging adults who smoke MJ and the continually decreasing age of MJ onset, these findings support the need and importance of early identification and intervention among individuals who do not report negative effects of MJ use.

In addition, while we did not find any statistically significant differences between the subject groups on any measure of clinical state or demographic variable, it is possible that the groups differed on measures that we did not assess. While some research has suggested that $\mathrm{MJ}$ smokers exhibit differences in personality factors relative to nonsmokers (Terracciano et al. 2008; Berg et al. 2011), which could potentially account for some of the heightened impulsivity observed in MJ smokers, the present sample of MJ smokers exhibited no significant differences in personality measures relative to control participants, as assessed by the NEO five-factor inventory (Costa and McCrae 1992), a well-validated personality inventory that provides scores in several domains, including openness, conscientiousness, agreeableness, extraversion, and neuroticism.

Finally, it remains unclear whether the reductions in FA in MJ smokers noted in the present study are the result of demyelination or damage to white matter, delayed or altered brain developmental patterns in MJ smokers, and if they precede or are the result of MJ use. Further, it is unclear if the alterations noted are reversible after extended periods of abstinence. In the current study, MJ smokers were asked to abstain from MJ use for a minimum of only $12 \mathrm{~h}$ in order to ensure they were not acutely intoxicated at the time of scanning. Future investigations should examine individuals with extended abstinence in order to determine what impact this has on measures of white matter microstructure. In 
addition, studies should also focus on former MJ smokers in order to determine the potential recovery of white matter integrity, as extended abstinence from MJ has been shown to result in a "normalization" of brain function (Sneider et al. 2009).

\section{Conclusions}

Findings from this study suggest that chronic, heavy MJ smokers have lower white matter fiber tract integrity relative to non-MJ-smoking participants and that earlier age of $\mathrm{MJ}$ onset is associated with lower FA levels. Further, MJ smokers demonstrated higher levels of reported impulsivity relative to control participants, which is related to FA levels, specifically within the early onset MJ group. Notably, lower FA levels are associated with higher reported impulsivity within this sample, suggesting a specific relationship between white matter organization and impulsivity in those individuals who begin smoking MJ prior to age 16. Taken together, these findings reinforce the idea that early onset of MJ use negatively impacts white matter development and is associated with behavioral impulsivity, a combination that may have enduring negative effects, particularly on the developing brain. Data from this study highlight the importance of early identification of MJ use among emerging adults and the need for efforts aimed at delaying or preventing the onset of MJ use.

Acknowledgments This project was supported by the National Institute of Drug Abuse 5 R21 DA021241 and 1R01 DA032646 awarded to Dr. Gruber.

Conflicts of interest All authors declare no conflicts of interest.

Open Access This article is distributed under the terms of the Creative Commons Attribution License which permits any use, distribution, and reproduction in any medium, provided the original author(s) and the source are credited.

\section{References}

Aboitiz F, Rodriguez E, Olivares R, Zaidel E (1996) Age-related changes in fibre composition of the human corpus callosum: sex differences. Neuroreport 7:1761-1764

Arnone D, Barrick TR, Chengappa S, Mackay CE, Clark CA, AbouSaleh MT (2008) Corpus callosum damage in heavy marijuana use: preliminary evidence from diffusion tensor tractography and tractbased spatial statistics. Neuroimage 41:1067-1074

Ashtari M, Cervellione K, Cottone J, Ardekani BA, Kurma S (2009) Diffusion abnormalities in adolescent and young adults with a history of heavy cannabis use. J Psychiatr Res 43:189-204

Bava S, Frank R, McQueeny T, Schweinsburg BC, Schweinsburg AD, Tapert SF (2009) Altered white matter microstructure in adolescent substance abusers. Psychiatry Res 173:228-237

Beck AT, Ward CH, Mendelson M, Mock J, Erbaugh J (1961) An inventory for measuring depression. Arch Gen Psychiatry 4:561571
Berg CJ, Buchanan TS, Grimsley L, Rodd J, Smith D (2011) Personality characteristics and health risk behaviors associated with current marijuana use among college students. Open J Prev Med 1:101-108

Bolla K, Rothman R, Cadet J (1999) Dose related neurobehavioral effects of chronic cocaine use. J Neuropsychiatry Clin Neurosci 11:361369

Brady KT, Myrick H, McElroy S (1998) The relationship between substance use disorders, impulse control disorders, and pathological aggression. Am J Addict 7:221-230

Bush G, Spencer TJ, Holmes J, Shin LM, Valera EM, Seidman MN, Surman C, Aleardi M, Mick E, Biederman J (2008) Functional magnetic resonance imaging of methylphenidate and placebo in attention-deficit/hyperactivity disorder during the multi source interference task. Arch Gen Psychiatr 65:102-114

Cha YM, White AM, Kuhn CM, Wilson WA, Swartzwelder HS (2006) Differential effects of delta9-THC on learning in adolescent and adult rats. Pharmacol Biochem Behav 83:448-455

Chaddock-Heyman L, Erickson KI, Voss MW, Powers JP, Knecht AM, Hillman CH, Kramer AF (2013) White matter microstructure is associated with cognitive control in children. Biol Psychiatry 94:109-115

Costa PT, McCrae RR (1992) NEO-PI-R professional manual. Revised NEO Personality Inventory (NEO-PIR) and NEO Five Factor Inventory (NEO-FFI). Psychological Assessment Resources, Odessa

Day AM, Metrik J, Spillane NS, Kahler CW (2013) Working memory and impulsivity predict marijuana-related problems among frequent users. Drug Alcohol Depend 131:171-174

Dougherty DM, Mathias CW, Dawes MA, Furr RM, Charles NE, Liguori A, Shannon EE, Acheson A (2013) Impulsivity, attention, memory, and decision-making among adolescent marijuana users. Psychopharmacology (Berl) 226:207-319

First MB, Spitzer RL, Gibbon M, Williams BW (1994) Structured clinical interview for axis I DSM IV disorder, patient edition (SCID-I/P) Version 2.0. Biometric Research Department, NY State Psychiatric Institute, New York

Glass M, Dragunow M, Faull RL (1998) Cannabinoid receptors in the human brain: a detailed anatomical and quantitative autoradiographic study in the fetal, neonatal and adult human brain. Neuroscience 77: 299-318

Gruber SA, Dahlgren MK, Sagar KA, Gonenc A, Killgore WDS (2012a) Age of onset of marijuana use impacts inhibitory processing. Neurosci Lett 511:89-94

Gruber SA, Sagar KA, Dahlgren MK, Olson DP, Centorrino F, Lukas SE (2012b) Marijuana impacts mood in bipolar disorder: a pilot study. Ment Health Subst Use 5:228-239

Gruber SA, Sagar KA, Dahlgren MK, Racine M, Lukas SE (2011a) Age of onset of marijuana use and executive function. Psychol Addict Behav 26:496-506

Gruber SA, Silveri MM, Dahlgren MK, Yurgelun-Todd D (2011b) Why so impulsive? White matter alterations are associated with impulsivity in chronic marijuana smokers. Exp Clin Psychopharmacol 3:231-242

Gruber SA, Yurgelun-Todd DA (2005) Neuroimaging of marijuana smokers during inhibitory processing: a pilot investigation. Brain Res Cogn Brain Res 23:107-118

Guy SM, Smith GM, Bentler PM (1994) Consequences of adolescent drug use and personality factors on adult drug use. J Drug Educ 24: 109-132

Hamilton M (1959) The assessment of anxiety states by rating. Br J Med Psychol 32:50-55

Harvey MA, Sellman JD, Porter RJ, Frampton DM (2007) The relationship between non-acute adolescent cannabis use and cognition. Drug Alcohol Rev 26:309-319

Heil SH, Johson MW, Higgins ST, Bicknel WK (2006) Delay discounting in currently using and currently abstinent cocaine-dependent outpatients and non-drug-using matched controls. Addict Behav $31: 1290-1294$ 
Hudkins M, O’Neill J, Tobias MC, Bartzokis G, London ED (2012) Cigarette smoking and white matter microstructure. Psychopharmacology (Berl) 221:285-295

Jacobsen LK, Picciotto MR, Heath CJ, Frost SJ, Tsou KA, Dwan RA, Jackowski MP, Constable RT, Mencl WE (2007) Prenatal and adolescent exposure to tobacco smoke modulates the development of white matter microstructure. J Neurosci 27: 13491-13498

Jacobus J, Bava S, Cohen-Zion M, Mahmood O, Tapert SF (2009a) Functional consequences of marijuana use in adolescents. Pharmacol Biochem Behav 92:559-565

Jacobus J, McQueeny T, Bava S, Schweinsburg BC, Frank LR, Yang TT, Tapert SF (2009b) White matter integrity in adolescents with histories of marijuana use and binge drinking. Neurotoxicol Teratol 31:349-355

Johnson MW, Bickel WK, Baker F, Moore BA, Badger GJ, Budney AJ (2010) Delay discounting in current and former marijuana-dependent individuals. Exp Clin Psychopharmacol 18:99-107

Johnston LD, O'Malley PM, Bachman JG, Schulenberg JE (2012) Monitoring the future national results on adolescent drug use: overview of key findings, 2011. Institute for Social Research, The University of Michigan, Ann Arbor

Lejuez CW, Aklin WM, Zvolensky MJ, Pedulla CM (2003) Evaluation of the Balloon Analogue Risk Task (BART) as a predictor of adolescent real-world risk-taking behaviours. J Adolesc 26: 475-479

Lejuez CW, Read JP, Kahler CW, Richards JB, Ramsey SE, Stuart GL, Strong DR, Brown RA (2002) Evaluation of behavioral measure of risk taking: the Balloon Analogue Risk Task (BART). J Exp Psychol Appl 8:75-84

Lim KO, Choi SJ, Pomara N, Wolkin A, Rotrosen JP (2002) Reduced frontal white matter integrity in cocaine dependence: a controlled diffusion tensor imaging study. Biol Psychiatry 51:890-895

Lisdahl KM, Price JS (2012) Increased marijuana use and gender predict poorer cognitive functioning in adolescents and emerging adults. $\mathrm{J}$ Int Neuropsychol Soc 18:678-688

Mata I, Perez-Iglesias R, Roiz-Santiañez R, Tordesillas-Gutierrez D, Pazos A, Gutierrez A, Jl V-B, Crespo-Facorro B (2010) Gyrification brain abnormalities associated with adolescence and early-adulthood cannabis use. Brain Res 41317:297-304

McHale S, Hunt N (2008) Executive function deficits in short-term abstinent cannabis users. Hum Psychopharmacol 23:409-415

Moeller FG, Hasan KM, Steinberg JL, Kramer LA, Dougherty DM, Santos RM, Valdes I, Swann AC, Barratt ES, Narayana PA (2005) Reduced anterior corpus callosum white matter integrity is related to increased impulsivity and reduced discriminability in cocaine-dependent subjects: diffusion tensor imaging. Neuropsychopharmacology 30: 610-617

Montgomery SA, Asberg M (1979) A new depression scale designed to be sensitive to change. Br J Psychiatry 134:382-389

Monti PM, Miranda R, Nixon K, Sher KJ, Swartzwelder HS, Tapert SF, White A, Crews FT (2005) Adolescence: booze, brains and behavior. Alcohol Clin Exp Res 29:207-220

Morriss MC, Zimmerman RA, Bilaniuk LT, Hunter JV, Haselgrove JC (1999) Change in brain water diffusion during childhood. Neuroradiology 41:929-934

Office of National Drug Control Policy (2012) Marijuana Resource Center. http://www.whitehouse.gov/ondcp/state-laws-related-tomarijuana. Accessed 1 Jul 2013

Pandya DN, Seltzer B (1982) Intrinsic connections and architectonics of posterior parietal cortex in the rhesus monkey. J Comp Neurol 204: 196-210

Park JH, Kim JJ, Lee SK, Seok JH, Chun J, Kim DI, Lee JD (2008) Corpus callosal connection mapping using cortical gray matter parcellation and DT-MRI. Hum Brain Mapp 29:503-516
Patton JM, Stanford MS, Barratt ES (1995) Factor structure of the Barratt Impulsiveness Scale. J Clin Psychol 51:768-774

Paul RH, Grieve SM, Niaura R, David SP, Laidlaw DH, Cohen R, Taylor G, Clark RC, Pogun S, Gordon E (2008) Chronic cigarette smoking and the microstructural integrity of white matter in healthy adults; a diffusion tensor imaging study. Nicotine Tob Res 10: 1370147

Pollock V, Cho DW, Reker D, Volavka J (1979) Profile of mood states: the factors and their physiological correlates. J Nerv Ment Dis 167: $612-614$

Pope HG, Yurgelun-Todd D (1996) The residual cognitive effects of heavy marijuana use in college students. JAMA 275:521527

Reise SP, Moore TM, Sabb FW, Brown AD, London ED (2013) The Barratt Impulsiveness Scale-11: reassessment of its structure in a community sample. Psychol Assess 25:631-642

Romero J, Garcia-Palermo E, Berrendero F, Garcia-Gil L, Hernandez ML, Ramos JA, Fernandez-Ruiz JJ (1997) Atypical location of cannabinoid receptors in white matter areas during rat brain development. Synapse 26:317-323

Salo R, Nordahl TE, Buonocore MH, Natsuaki Y, Waters C, Moore CD, Galloway GP, Leamon MH (2009) Cognitive control and white matter callosal microstructure in methamphetamine dependent subjects: a diffusion tensor imaging study. Biol Psychiatry 65:122128

Schneider M (2008) Puberty as a highly vulnerable development period for the consequences of cannabis exposure. Addict Biol 13:253263

Schneider M, Koch M (2003) Chronic pubertal, but not adult chronic cannabinoid treatment impairs sensorimotor gating, recognition memory, and the performance in a progressive ratio task in adult rats. Neuropsychopharmacology 28:1760-1769

Schweinsburg AD, Nagel BJ, Schweinsburg BC, Park A, Theilmann J, Tapert SF (2008) Abstinent adolescent marijuana users show altered fMRI response during spatial working memory. Psychiatry Res 163: $40-51$

Sneider JT, Pope HG, Silveri MM, Simpson NS, Gruber SA, YurgelunTodd DA (2009) Differences in regional blood volume during a 28day period of abstinence in chronic cannabis smokers. Eur Neuropsychopharmacol 18:612-619

Solowij N, Pesa N (2010) Cognitive abnormalities and cannabis use. Rev Bras Psiquiatr 32:S31-S40

Steinberg L, Sharp C, Stanford MS, Tharp AT (2013) New tricks for an old measure: the development $f$ the Barratt Impulsiveness ScaleBrief (BIS-Brief). Psychol Assess 25:216-226

Spielberger CD, Gorsuch RL, Lushene PR, Vagg PR, Jacobs AG (1983) Manual for the state-trait anxiety inventory. Consulting Psychologists Press, Palo Alto

Substance Abuse and Mental Health Services Administration (2012) Results from the 2011 National Survey on Drug Use and Health: Summary of National Findings. NSDUH Series H-44. HHS Pub. No. (SMA) 12-4713. SAMHSA, Rockville

Tapert SF, Schweinsburg AD, Drummond SPA, Paulus MP, Brown SA, Yang TT, Frank LR (2007) Functional MRI of inhibitory processing in abstinent adolescent marijuana users. Psychopharmacology (Berl) 194:173-183

Terracciano A, Lockenhoff CE, Crum RM, Bienvenu OJ, Costa PT (2008) Five-factor model personality profiles of drug users. BMC Psychiatry 8:22

Vangsness L, Bry BH, LaBouvie EW (2005) Impulsivity, negative expectancies, and marijuana use: a test of the acquired preparedness model. Addict Behav 30:1071-1076

Vitaro F, Gendreau PL, Tremblay RE, Oligny P (1998) Reactive and proactive aggression differentially predict later conduct problems. J Child Psychol Psychiatry 39:377-385 
Watson D, Clark LA, Tellegen A (1988) Development and validation of brief measures of positive and negative affect: the PANAS scales. J Pers Soc Psychol 54:1063-2070

Wechsler D (1999) Wechsler abbreviated scale of intelligence. The Psychological Corporation, San Antonio

Young RC, Biggs JT, Ziegler VE, Meyer DA (1978) A rating scale for mania: reliability, validity and sensitivity. Br J Psychiatry 133:429435
Yucel M, Solowij N, Respondek C, Whittle S, Fornito A, Pantelis C, Lubman DI (2008) Regional brain abnormalities associated with long-term heavy cannabis use. Arch Gen Psychiatry 65:694701

Zalesky A, Solowij N, Yucel M, Lubman DI, Takagi M, Harding IH, Lorenzetti V, Wang R, Searle K, Pantelis C, Seal M (2012) Effect of long-term cannabis use on axonal fibre connectivity. Brain 135: 2245-2255 